\title{
Correction to: Telomere length of gallbladder epithelium is shortened in patients with congenital biliary dilatation: measurement by quantitative fluorescence in situ hybridization
}

\author{
Yuto Aoki $^{1,2}$ (D) Junko Aida ${ }^{2}$-Youichi Kawano ${ }^{2,3}$ - Ken-ichi Nakamura ${ }^{2}$. \\ Naotaka Izumiyama-Shimomura ${ }^{2}$ - Naoshi Ishikawa ${ }^{2}$ Tomio Arai ${ }^{4}$. \\ Yoshiharu Nakamura $^{1}$ - Nobuhiko Taniai ${ }^{1} \cdot$ Eiji Uchida $^{1} \cdot$ Kaiyo Takubo $^{2}$. \\ Toshiyuki Ishiwata ${ }^{2}$
}

Published online: 5 December 2017

(c) Japanese Society of Gastroenterology 2017

\section{Correction to: J Gastroenterol}

https://doi.org/10.1007/s00535-017-1411-5

In the original publication of this article, Fig. 5 was published with incorrect color of the approximate lines of CBD and Control.

The corrected figure is given in the following page.

The original article can be found online at https://doi.org/10.1007/ s00535-017-1411-5.

Yuto Aoki

yutoaoki@nms.ac.jp

$\bowtie$ Toshiyuki Ishiwata

tishiwat@tmig.or.jp

1 Department of Gastrointestinal and Hepato-Biliary-

Pancreatic Surgery, Nippon Medical School, 1-1-5 Sendagi,

Bunkyo-ku, Tokyo 113-8603, Japan

2 Research Team for Geriatric Pathology and Department of Pathology, Tokyo Metropolitan Geriatric Hospital and Institute of Gerontology, Tokyo, Japan

3 Department of Surgery, Nippon Medical School Chiba Hokusoh Hospital, Chiba, Japan

4 Department of Pathology, Tokyo Metropolitan Geriatric Hospital, Tokyo, Japan 

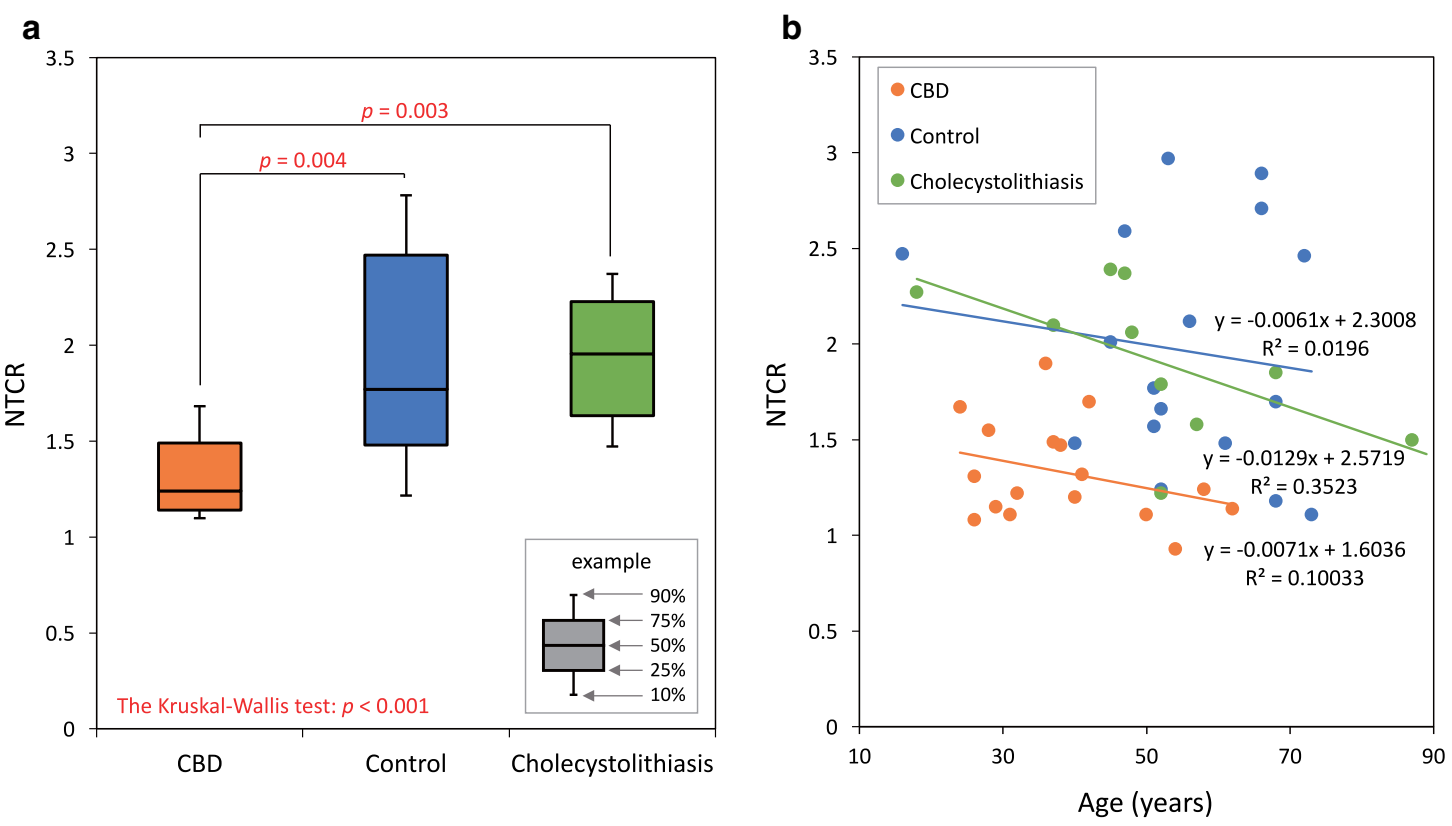